\title{
THE PREVENTION OF PLANTAR ULCER IN LEPROSY
}

\author{
By E. W. Price, M.D., F.R.C.S.E.
}

The frequency of plantar ulcer is such that, in many places, new cases occur more rapidly than medical care can heal and prevent the recurrence of existing ones. A method of prevention is essential if progress is to be made.

There are several possible approaches to the problem, and their study needs to be correlated with the subsequent ulcer-history of the foot. They can be undertaken by anyone engaged in the care of leprosy patients and, with the exception of those for vasomotor disturbance, no special equipment is used. The purpose of this paper is to encourage interest in one or other method, so as to increase our knowledge of the clinical value of each technique.

\section{Types of Plantar Ulcer}

An important distinction must be made between the pliant foot and the rigid foot; the ulcers appearing on each type are of different etiology and are not likely to respond to similar treatment. The difference between the two types may be obvious, as between the foot of the healthy boy,and that of an elderly person with subtalar arthritis; but in case of doubt, it can be demonstrated by asking the patient to rise on tip-toe. The ability to do this and the shape of the foot in this position will make the state of the foot apparent (Fig. 1).

The pliant foot will show the 'dynamic type' of plantar ulcer, on the points of the sole, where the stresses of walking cause the breakdown in the protective mechanisms of the neuropathic foot. (Lep. Review 1959, 30, 98.)

Measures which control these stresses (e.g. rigid-sole footwear) are likely to control the occurrence and recurrence of the lesion.

On the contrary, the rigid foot is more likely to show the 'static type' of ulcers, which are pressure lesions at whichever part of the deformed foot is most prominent in the sole. The rigidity of the foot is due to the scarring of chronic infection and injury, and to infective or degenerative arthritis. Footwear may be designed to redistribute weight as local circumstances permit; but many cases become candidates for amputation.

\section{A Notation for Plantar Ulcers of the Pliant Foot}

The ulcers to be studied are of the 'dynamic type' and a rapid method of recording the lesion is essential for accuracy of comparison. The following has been used for some years and is found to be satisfactory (Fig. 2.) 
The Tip-Toe Test of Foot-Type. Fig. 1.

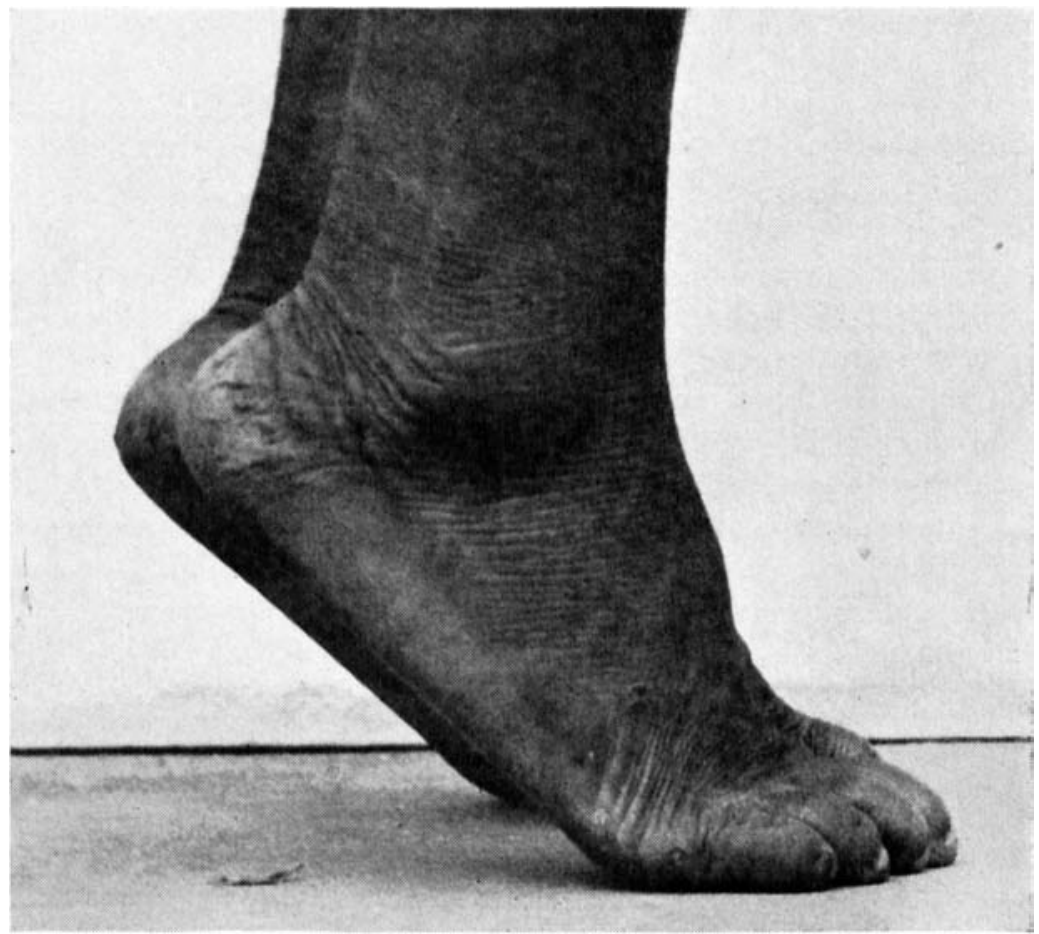

(a) The Pliant FoOt. The body rises on 'tip-toe' on to the heads of the metatarsals. If this foot ulcerates, the ulcer will be of the 'dynamic' type.

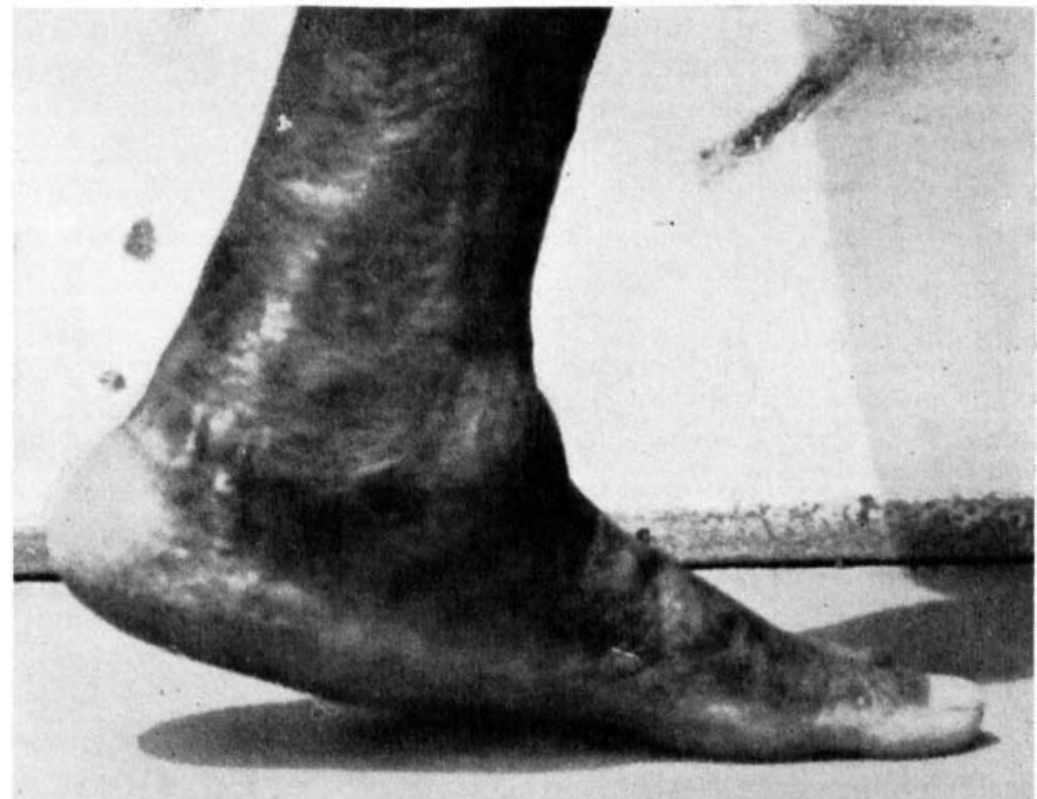

(b) THE RIGID FOOT. The foot with scarred plantar tissues, or with diseased bones and joints, is unable to rise on 'tip-toe'. Plantar ulcers of this foot are of 'static' type and are traumatic wounds on whichever part of the sole is most prominent. 
Notation for Dynamic Plantar Ulcers. Fig. 2

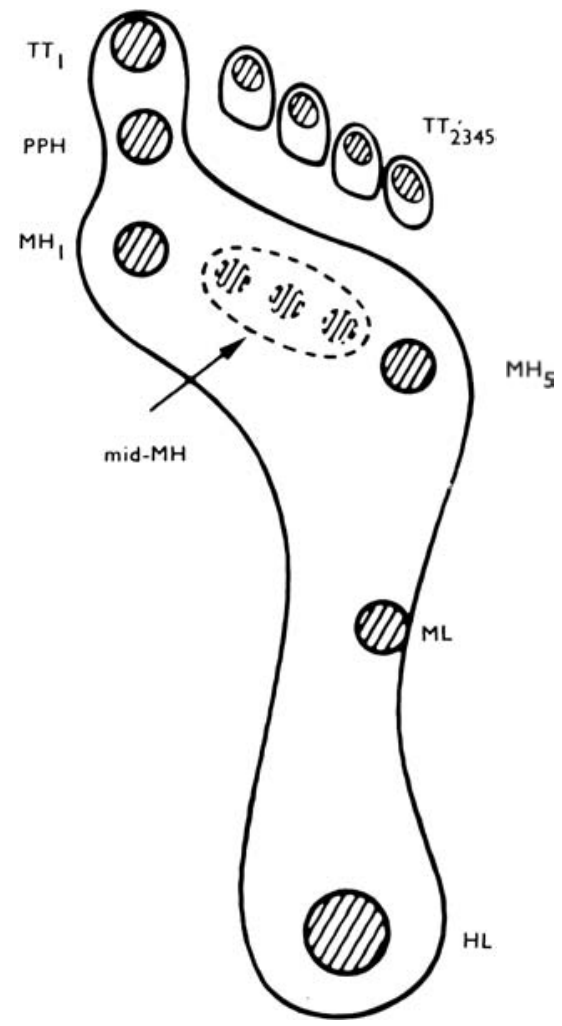

Letters refer to underlying bony prominence:

$\mathrm{TT}=$ toe-tip

$\mathrm{PPH}=$ proximal phalangeal head of big toe

$\mathrm{ML}=$ mid-lateral

$\mathrm{HL}=$ heel

$\mathrm{MH}=$ metatarsal head

Multiple ulcers on lateral toes are reckoned as a single ulcer spread over more than one toe.

1. The ulcers at impact (heel) and push-off (toe tips) are denoted by $\mathrm{HL}$ and TT.

2. The ulcers at points of plantar flexion are indicated by reference to the underlying bony prominence: $\mathrm{MH}_{1-5}, \mathrm{PPH}$; or as ML (mid-lateral) in the case of the tuberosity of the fifth metatarsal.

Reference is also made to the side ( $\mathrm{R}$ or $\mathrm{L}$ ), and to the diameter of the ulcer, e.g. $\mathrm{RMH}_{1} 2 \mathrm{cms}=$ an ulcer $0 \cdot 2 \mathrm{~cm}$. large under the first metatarsal head of the right foot.

Toe tip ulcers are often multiple, and are recorded as occurring on the big-toe $\left(\mathrm{TT}_{1}\right)$ or on the lateral toes $\left(\mathrm{TT}_{2345}\right)$. Multiple ulcers of the lateral toes are counted as one ulcer spread over several toes. The true plantar ulcer of the toe tip must be distinguished from the terminal ulcer sometimes seen in claw-toes, and from the dorsal ulcer of some cases of foot-drop.

The common ulcer under the interphalangeal joint of the big toe 
(PPH) may in special cases (e.g. loss of big toe) be replaced by one under the proximal inter-phalangeal joint of the second toe and is then recorded as $\mathrm{PPH}_{2}$.

\section{Methods of Study}

Possible means of knowing that plantar ulcer is about to develop are:

1. Recognition of early sensory loss.

2. Recognition of early motor loss.

3. Recognition of vasomotor disturbance.

4. Clinical recognition of the pre-ulcerative state.

Stress is laid on the necessity for early recognition. To await complete anaesthesia, obvious foot drop or incipient infection of a callosity is known to be too late. The methods proposed here are designed to detect early loss of neural function.

(A) Tests for mis-localisation of pressure

In early attempts to recognise pre-ulcerative feet, it was soon found that the criterion of anaesthesia to pain (e.g. test by pin prick) did not reveal some feet that in the event finally ulcerated. Attention has been drawn to this phenomenon by LANGUILLON et al (1960), but its significance is not yet known. An earlier stage of sensory loss must be sought, and one such is that of mis-localisation of pressure (MLP).

Recent work (WEDDELL, 1962) has shown that each area of skin is supplied by axons which may enter the spinal cord by as many as four dorsal roots.

Accurate localisation depends on the integrity of these pathways but as many as $25 \%$ may be destroyed without clinical evidence of loss. Further denervation results in a state when the patient is aware he is being touched but is unable to localise accurately. This state offers a field of study that may reveal a significant relation to pending ulceration. Tests already done show that, for each neuropathic foot that is not completely anaesthetic, there is a threshold pressure below which localisation is faulty or absent, but above which recognition with or without accurate localisation is present. This threshold area needs proper investigation with reference to future plantar ulceration.

Before attempting the test on abnormal feet, the reaction of normal feet in the region under examination should be learnt by experience. The test is then done as follows:

The ulcer-bearing area of the sole is systematically stimulated at random using a nylon thread which projects for $1 \mathrm{~cm}$. from a holder. Pressure is increased until the thread yields. The patient (blindfold) then localises each spot with his finger. Accurate localisation is considered normal.

It has been found wise to allow the patient to do the test first with 
The Kindetograph or Dynamic Foot-Print (after Morton). Fig. 3

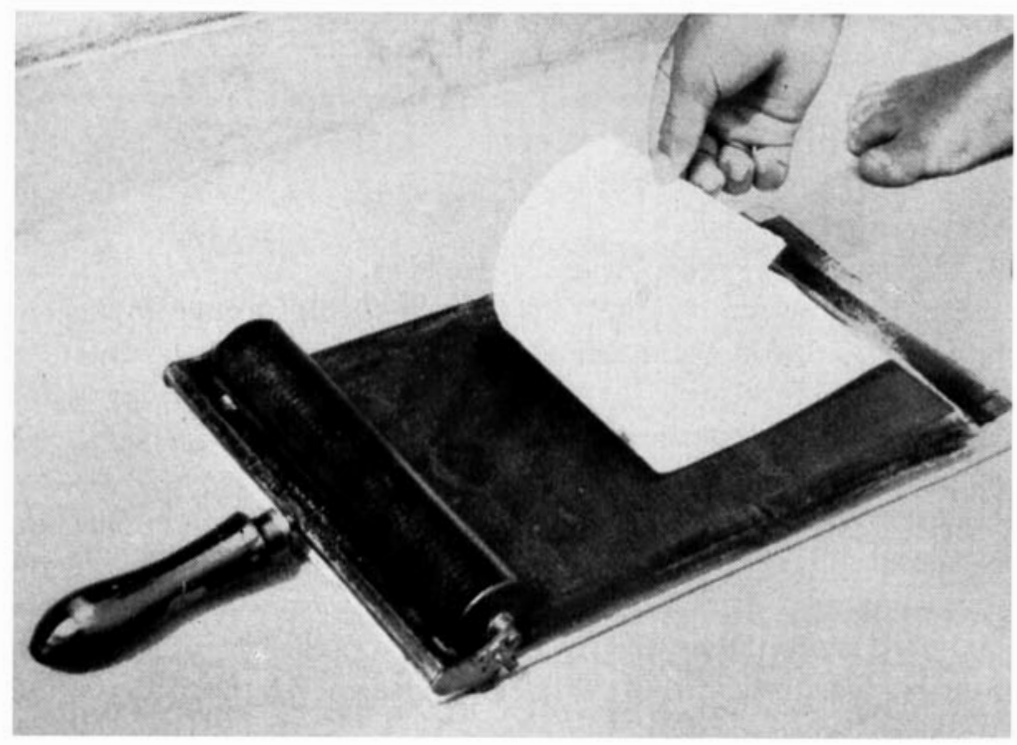

(a) THE APPARATUS. A thin rubber mat with compressible corrugations or scraperboard with embossed pattern is inked with a roller, as in the figure. This is then removed and the mat is covered with paper.

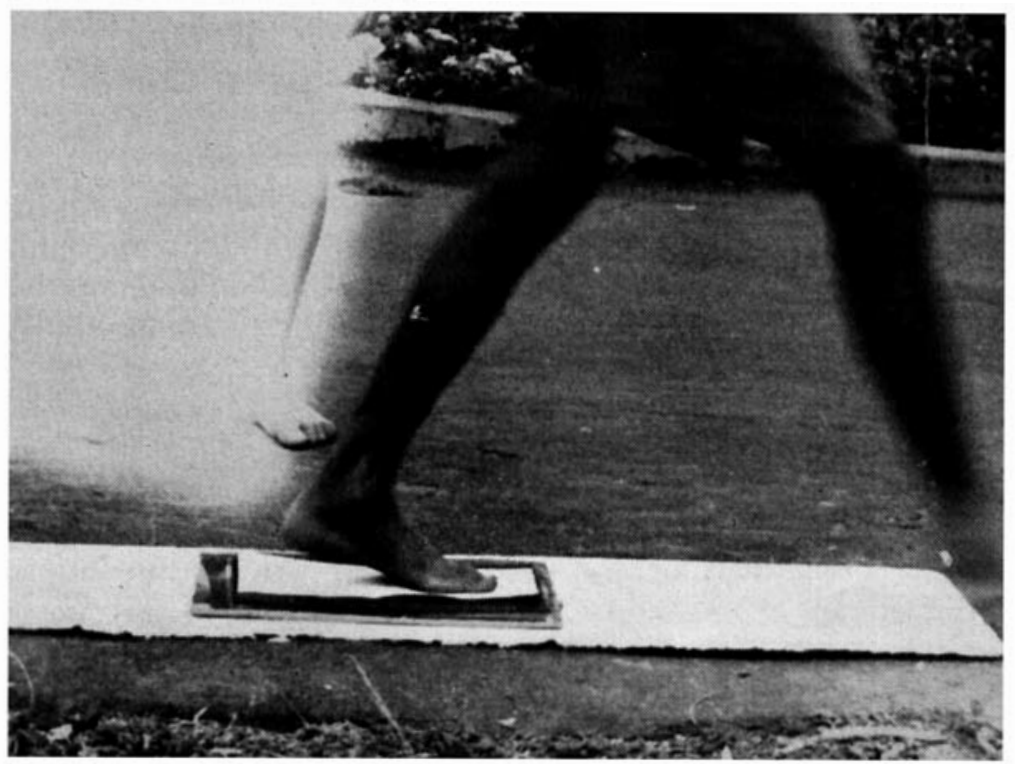

(b) THE Method of UsE. The patient walks across it and the resulting imprint is the total of the sequence of pressures from heel to toes as the step is taken. Imprints are shown in Fig. 4. 
the eyes open and then to cover the eyes completely, and not rely on his shutting his eyes, or covering them with his hand.

The results should be recorded on a diagram of the sole, and should include the pressure used; this can be found by using the same pressure on the pan of a laboratory balance. Cases with loss of localisation should be followed serially without treatment, repeating the test at monthly intervals, to see whether or not ulceration occurs subsequently.

(B) The 'kinetograph' or Dynamic foot-print

Early loss of motor innervation can be demonstrated by the use of the foot-print of the walking foot. The pressures represented by the 'dynamic' foot-print (DFP) are the resultant of muscular action on toes and foot, together with the weight of the body in motion. Disturbance of this balance is seen as an abnormal DFP.

The normal DFP shows that after initial impact at the heel, the pressure passes rapidly to the head of the 5th metatarsal, clearing the intervening area. It then proceeds across the metatarsal pads to its maximum under the first head, and then proceeds forward to the tip of the big toe, also clearing the intervening area. All the toes combine for the final push-off.

The dynamic foot-print is obtained by the use of a rubber mat or MORTON board such as artists' scraper-board (LAKE) on which a fine geometric pattern is embossed.

The print is taken as follows: (Fig. 3)

The mat is inked with printer's ink using a roller. A sheet of absorbent paper, such as newsprint, is laid lightly on top, and the patient walks barefoot across it, stepping on the paper with one foot as he continues onwards.

The mat is then re-inked and the other foot treated similarly. The prints are allowed to dry and are then added to the records. It is important to re-ink between each test, to protect the mat from dust after use, and to note that the print is that of the opposite side to what it first appears to be. As with other tests, familiarity with the normal is essential (Fig. 4a).

Interpretation of Foot-prints

The common deviations of interest are:

(i) Lateral displacement of maximal metatarsal-pad pressure (Fig. 4b).

The normal maximum at $\mathrm{MH}_{1}$ may be displaced to $\mathrm{MH}_{2}$ or even more laterally. Some displacement is normal if the patient uses an intoeing gait ('pigeon-toes'), but otherwise, it indicates failure of the pronators of the foot.

(ii) Appearance of mid-lateral pressure (Fig. 4b).

The appearance of an area of pressure mid-laterally between heel and fifth metatarsal pad represents early peroneal failure and is common with the previous abnormality. 
(iii) Appearance of pressure area at base of big-toe (Fig 4c).

Pressure at the base of the big toe under the inter-phalangeal joint represents weakness of the extensor hallucis longus.

(iv) Areas of concentrated pressure (Fig. 4d).

These are common at the metatarsal pads, the proximal phalangeal head of the big toe and the base of the fifth metatarsal. They represent failure of intrinsic musculature, and are often precursors of ulceration.

(v) 'Clutching' toes and 'cocked' toes (Fig. 4c).

These examples of muscle spasm due to nerve irritation, and of palsy are appropriately indicated by the DFP, and may precede ulceration of toe tips.

It must always be recalled that other conditions than leprosy can upset the normal balance of the foot. None of the above abnormalities alone represent a lesion of leprosy; but taken with other signs, they may be highly significant.

It will be found that some patients show similar areas of increased pressure on both feet; yet only one side ulcerates. There are evidently additional factors to be elucidated.

(C) Tests for Vasomotor Dysfunction

The tests for vasomotor dysfunction include those based on variations of skin temperature and usually undertaken in centres of research. These lesions have been fully investigated by several workers such as GoKHALE et al (1959) but the results are inconclusive other than demonstrating that there is a loss of vasomotor control in leprosy.

In view of the special apparatus needed, test for vasomotor loss are unlikely to be of wide application in local centres of leprosy treatment and are theref ore omitted from this study.

(D) Clinical Recognition of the Pre-ulcerative State

The early signs and symptoms of the pre-ulcerative state are described in Lep. Review 1959, 30, 180. Further evidence is the condition of 'shiny pads'; the metatarsal pads and toe-tips appear polished and burnished, due to repeated friction with the ground at each step, as the forefoot slips slightly forward at maximal bodyweight. This condition discloses failure of the toes to grip the ground and represents early failure of the intrinsic musculature.

It is not always realised that the pre-ulcerative state, of which the signs may be localised plantar swelling and tenderness, splaying of toe, necrosis blister, and shiny pads, is only likely to be seen when weekly foot-inspection is practised. The cycle of events from early deep tissue damage to frank ulcer may occur in ten days, and even fortnightly inspections miss many cases. In a recent series of cases carefully observed for periods up to 18 months in Eastern Nigeria, this state was noted at sometime or other in about half the cases under observation for the pre-ulcerative state; a few displayed it on 


\section{Dynamic Foot-Prints (DFP). Fig. 4}

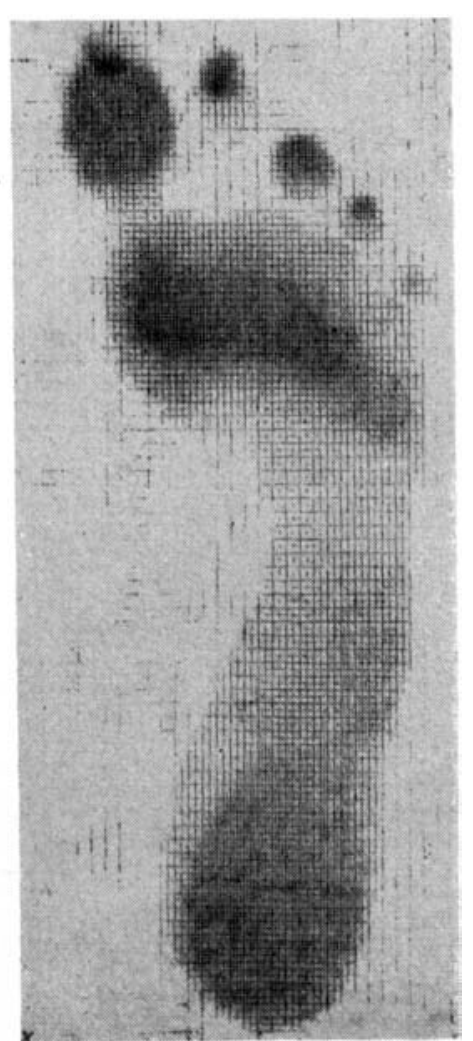

a.

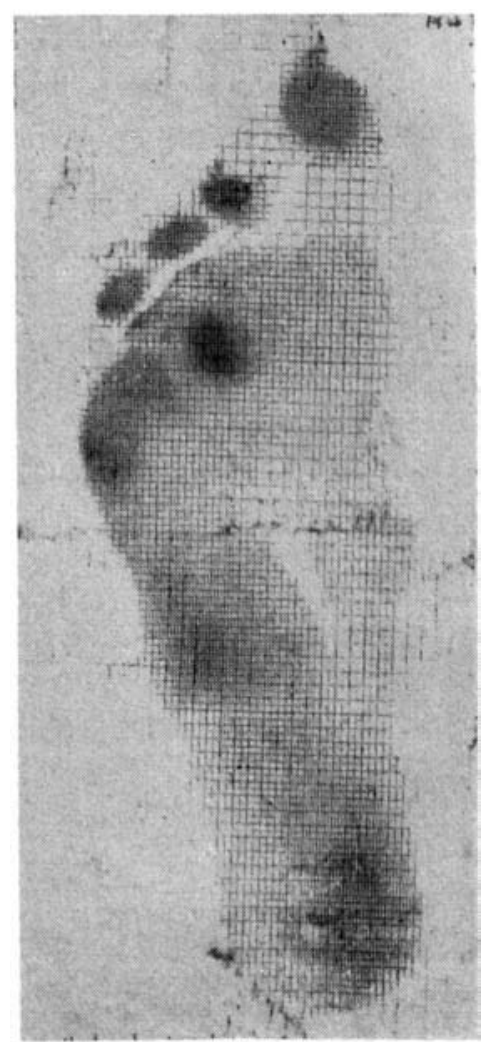

b.

These are obtained by a sequence of imprints beginning at the heel, and passing in sequence to the 5th MT pad, across to the 1st MT pad, and then to the final push-off in which all toe-tips normally co-operate.

(a) Normal. Note the gap between heel and 5th MT pad, and between 1st MT pad and 1 st toe-tip. Maximal pressure is at 1 st MT pad. .

(b) Pronator Weakness. Maximal pressure shifts laterally on the MT pads, and mid-lateral pressure appears. Lateral shift on MT pads by itself may be due to an in-toeing gait ('pigeon toes').

several occasions. In each case, ulceration was avoided by complete bed rest from ten to twenty days without other treatment.

Clinical evidence of the pre-ulcerative state is the last opportunity to avoid ulceration. Failure to recognise it penalises the patient by the development of an open ulcer, which may last for months or years if inadequately treated. It is repeated that the above condition is only likely to occur in the pliant foot.

\section{Discussion}

It is likely that multiple factors are responsible for the moment of ulceration in a pliant neuropathic foot, though the dominant role of the traumatic effect of walking appears to be established. The 


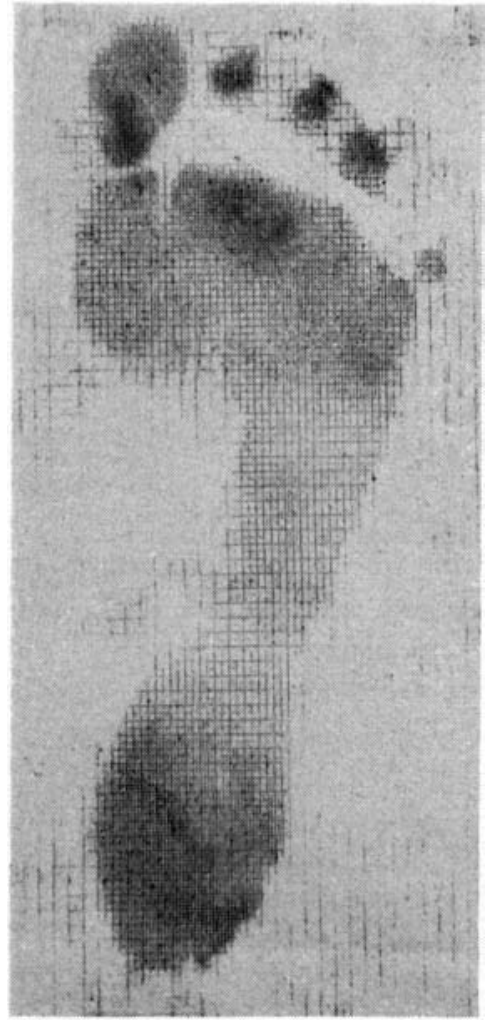

c.

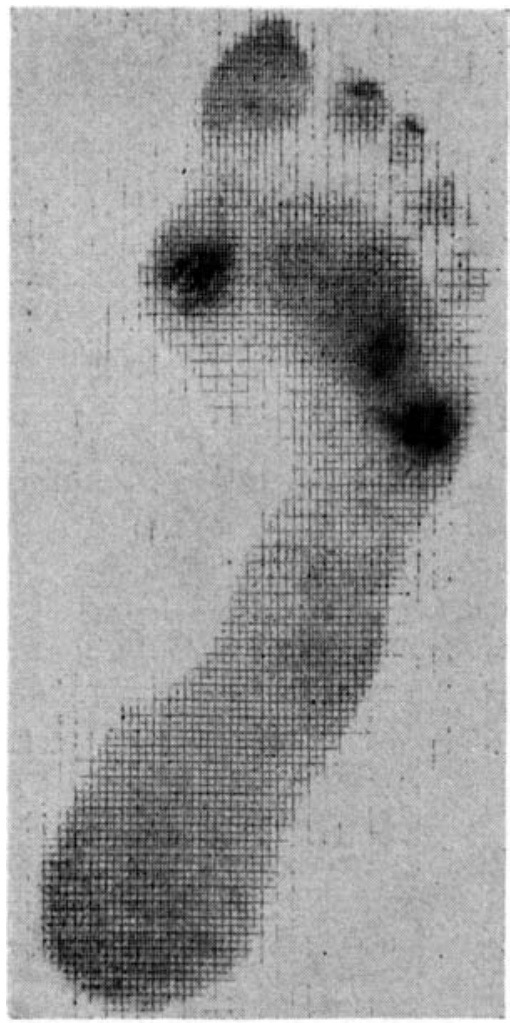

d.

(c) Failure of Big Toe Control. Pressure appears between 1 st MT pad and big toe and may be the maximal. It represents failure of big toe extensors or spasm of the flexors. Note the 'clutching' of 3rd and 4th toes, and the 'cocking' of 5th toe, due to intrinsic disturbance.

(d) Localised INCREASE of PRESSURE. The extreme pressures on pads of $M T_{1}$ and $M T_{5}$ are obvious and represent intrinsic failure of these digits. They often precede ulceration and are an important danger signal.

immediate need is for a method of recognising clinically, or by simple tests usable in rural conditions, when a foot is likely to ulcerate or is on the point of doing so. The latter need appears to be met by the recognition of the preulcerative state which precedes ulceration by less than ten days. None of the tests described here is found to disclose by itself the foot which is likely to ulcerate, but it is possible that a combination of tests and clinical observation may be adequate.

Apart from the obvious advantage of knowing that a foot is in the danger period - and when, conversely, it has passed out of that period - there is the practical advantage of avoiding unnecessary, and often monotonous, foot-inspection and the provision of footwear that will have been unnecessary.

In view of the number of workers who see patients at close quarters in leprosaria, and who are ideally placed for following 
clinical events and ulceration, it is hoped that this paper will encourage the careful testing and observation of a series against an equivalent number of control cases who are simply observed without testing or preventive treatment. In this way; the value of each method will be apparent.

\section{Summary}

1. The importance of prevention of plantar ulceration is stressed.

2. A distinction is drawn between 'dynamic' and 'static' types of plantar ulcer.

3. Methods of determining an early state of neural loss are defined with a view to clinical trials of their value in the prevention of ulcer.

4. Among the multiple factors that precipitate ulceration are noted: sensory loss, motor loss, vasomotor loss, lack of footinspection, lack of co-operation from the patient.

The rubber mats can be obtained from Down Bros. Ltd., 70 Grenville Street, Toronto 5, Ontario, Canada (U.S. \$8 each); and scraper-board can be obtained from Winsor \& Newton Ltd., Wealdstone, Harrow, Middlesex, England. A size and quality suitable are No. 17 white, 19 in. by 12 in. which costs 4 s. (U.S. $\$ 0.60$ ) a sheet, and will provide two pieces, available two days use each, for any number of patients on one day before it is finally discarded.

\section{References}

Gokhale, B. B., Vable, S. M. and Modak, S. (1959), Lep. Review 1959, 30, 234.

Languillon, J., Bourrel, P., Boissan, R. H. and Picard, P., Med. Trop. 1960, 20, 219.

Weddell, G. and Miller, S., Amer. Rev. Physiology 1962, 24, 199. 